\title{
Rôles du microARN miR-155 dans les démences associées au syndrome de Down
}

Gerard Nuovo ${ }^{1,2}$, Esmerina Tili ${ }^{3,4}$, Hamdy Awad ${ }^{3}$, Jean-Jacques Michaille ${ }^{5}$

Origine génétique et physiopathologie du syndrome de Down

Le syndrome de Down (SD) ${ }^{1}$ correspond à l'anomalie chromosomique la plus fréquente chez l'homme, avec environ six millions de personnes affectées dans le monde [1]. Chaque année, environ un bébé sur 700 naît porteur de ce syndrome. La fréquence de ce désordre chromosomique s'accroît avec l'âge de la mère, avec une forte augmentation à l'approche de la quarantaine. Près de $50 \%$ des bébés affectés par le SD présentent des anomalies cardiaques congénitales, dont un grand nombre requiert une intervention chirurgicale. Par rapport aux enfants non porteurs, les enfants SD présentent un risque plus élevé de présenter des problèmes auditifs ou oculaires, d'apnée du sommeil, du métabolisme, d'anémie, de luxation de la hanche, de comportement, et de développer une leucémie du jeune enfant.

Les anomalies chromosomiques responsables du SD impliquent toutes le chromosome 21. Ce petit chromosome comporte 48 millions de paires de bases et représente de 1,5 à $2 \%$ de I'ADN total de la cellule. Dans environ $95 \%$ des cas, le SD est dû à une trisomie 21 avec présence dans toutes les cellules de l'individu d'un troisième chromosome 21 par suite de non-disjonction au cours de la méiose I ou II. Mais d'autres anomalies plus rares sont parfois présentes. La présence d'une copie surnuméraire suggère que tous les gènes du chro-

\footnotetext{
${ }^{1}$ Autrefois appelé trisomie 21.
}

mosome 21 puissent être surexprimés chez les individus porteurs du SD. En fait, seulement $30 \%$ environ d'entre eux le sont, l'expression des autres gènes étant, soit compensée par des effets épigénétiques, soit hautement variable d'un individu à l'autre.

\section{SD et démence sénile}

L'augmentation de la fréquence de la maladie d'Alzheimer (MA) chez les individus SD a suivi celle de leur espérance de vie à la naissance [2] $(\rightarrow)$. La MA est une pathologie liée à l'âge qui

$(\rightarrow)$ Voir la Synthèse de J.M. Delabar $\mathrm{m} / \mathrm{s}$ $n^{\circ} 4$, avril 2010, page 371

s'accompagne de la dégénérescence fonctionnelle et de la mort progressive des neurones. Elle est la maladie neurodégénérative la plus fréquente dans les pays développés, avec plus de 46 millions de cas identifiés dans le monde. La MA est la cause principale d'apparition des démences séniles, et entraîne un déclin progressif des fonctions cognitives affectant en premier lieu la mémoire. Elle s'accompagne d'une perte progressive de la capacité à s'exprimer et de changements du comportement et conduit à la mort à plus ou moins long terme [3].

Les adultes atteints de SD présentent un risque accru de développer une maladie d'Alzheimer, et en particulier la forme «précoce » qui apparaît avant l'âge de 65 ans. Le diagnostic de MA chez ces personnes n'est pas des plus aisés, du fait de leurs capacités intellectuelles généralement réduites, de la forte variabilité de leurs performances dans
${ }^{1}$ GNOME Diagnostics, Powell, OH 43065, ÉtatsUnis.

${ }^{2}$ Comprehensive Cancer Center, The Ohio State University, Wexner Medical Center, Columbus, $\mathrm{OH} 43210$, États-Unis.

${ }^{3}$ Department of Anesthesiology, The Ohio State University, Wexner Medical Center, Columbus, $\mathrm{OH} 43210$, États-Unis.

${ }^{4}$ Department of Cancer Biology and Genetics, The Ohio State University, Wexner Medical Center, Columbus, $\mathrm{OH} 43210$, États-Unis.

${ }^{5}$ BioPerox-IL, Université de Bourgogne-

Franche Comté, Faculté des Sciences Gabriel, 6 Bd. Gabriel, 21000 Dijon, France.

Jean-Jacques.Michaille@u-bourgogne.fr

les tests cognitifs, et de la nécessité de s'appuyer en partie sur les observations des personnes en charge de ces patients pour estimer l'impact de cette pathologie sur leurs interactions sociales [3]. La prévalence de la MA chez les personnes présentant un SD varie de 7 à $50 \%$ selon les études. Cette prévalence augmente fortement avec l'âge: $11 \%$ entre 40 et 49 ans, $66 \%$ entre 50 et 59 ans, $77 \%$ entre 60 et 69 ans, et $100 \%$ chez les individus de 70 ans ou plus $[3,4]$.

Aux âges avancés, la plupart des personnes souffrant de SD présentent des caractéristiques pathologiques similaires à celles que l'on observe dans la MA, à savoir l'accumulation extracellulaire progressive de plaques de protéine $\beta$-amyloïde $(\beta A)$, et celle, intraneuronale, de neurofibrilles formées par un enchevêtrement de dimères de protéine Tau (tubulin-associated unit) [4]. Les plaques de $\beta$ A compriment et endommagent les neurones, induisant un stress oxydant qui conduit à l'activation de la microglie (les cellules immunitaires résidentes du cerveau) et finalement à la mort neuronale par apoptose ou nécrose. Ces plaques sont formées par l'accumulation d'une forme anormale de $\beta A$ contenant 42 acides aminés. Cette dernière est produite par clivage anormal de I'APP (amyloïd protein precur- 


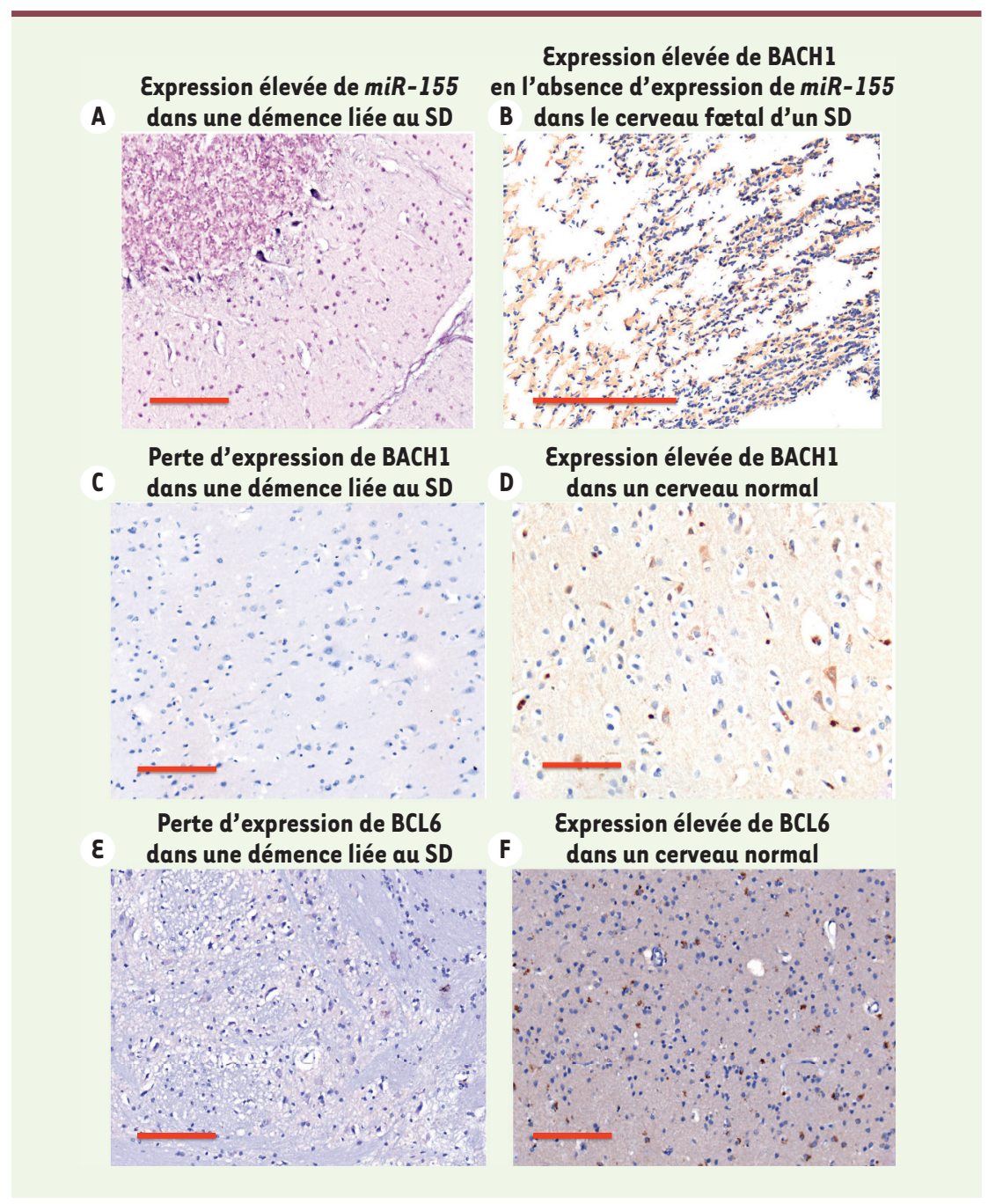

Figure 1. Détection de miR-155, BACH1 et BCL6 sur coupes de cerveau. L'élévation du niveau d'expression de miR-155 diminue celui des protéines BACHl (répresseur transcriptionnel) et BCL6 (facteur pro-survie), toutes deux codées par des ARN messagers cibles de ce microARN. A. Détection de miR-155 (en bleu) par hybridation in situ sur coupes de cerveau d'un individu ayant souffert d'une démence associée au syndrome de Down (SD). La contre-coloration au «nuclear fast red» donne une couleur rose. B-F. Détection des protéines BACHI et BCL6 par immunohistochimie sur coupes de cerveau d'un fœtus porteur du SD (B), d'un individu adulte ayant souffert d'une démence associée au SD ( $C$ et $\varepsilon$ ), ou d'un adulte sain ( $D$ et $F$ ). Les protéines sont colorées en brun, la contre-coloration à l'hématoxyline donne une couleur bleue. Longueur des barres : $150 \mu \mathrm{m}$.

sor), le précurseur de la $\beta A$. Le gène $A P P$, situé sur le chromosome 21 , est présent en trois exemplaires chez les SD. Les niveaux de $\beta A$ sont ainsi plus élevés chez les SD jeunes que chez les individus non porteurs de la trisomie, et la concentration de $\beta$ A augmente de façon exponentielle après 40 ans chez ces personnes. La protéine Tau interagit avec la malement élevée de Tau diminue son affinité pour les microtubules et conduit à la formation d'enchevêtrements de dimères de filaments hélicoïdaux et de filaments droits produisant les neurofibrilles caractéristiques de la maladie d'Alzheimer. Au final, ces changements moléculaires induisent des changements dans l'anatomie du cerveau qui sont retrouvés dans les démences induites par la MA, chez les individus SD comme chez les individus non SD [5].

\section{Les microARN présents sur le chromosome 21 humain}

Les microARN sont de petits ARN non codants qui régulent la stabilité et/ ou la traduction des transcrits dont ils ciblent habituellement la partie 3'-non traduite par l'intermédiaire de séquences consensus spécifiques. Ce sont des régulateurs extrêmement puissants pouvant moduler directement, selon le cas, le niveau d'expression de plusieurs dizaines à plusieurs centaines d'ARNm (ARN messagers), et, indirectement, celui d'un nombre beaucoup plus élevé de gènes par le fait que certains des transcrits qu'ils ciblent codent des facteurs de transcription, des facteurs impliqués dans diverses voies de transduction de signal, ou encore des enzymes impliquées dans le contrôle épigénétique de l'expression des gènes. Les microARN jouent un rôle clé dans le maintien de l'homéostasie cellulaire, et les pathologies majeures comme les cancers, les maladies cardiovasculaires, neurodégénératives, métaboliques ou auto-immunes ont toutes été liées à des anomalies d'expression de certains microARN. En particulier, le rôle des microARN dans le développement ou l'aggravation de la MA est maintenant bien établi [6].

Le chromosome 21 porte des gènes de microARN, parmi lesquels un groupe de 3 microARN (miR-let7c, miR-99a et miR-125b) et deux microARN isolés, miR-155 et miR-802, tous impliqués à des degrés divers dans la régulation du fonctionnement du cerveau [4] et 
surexprimés dans le cerveau des personnes présentant une trisomie 21 [7]. miR-155 est l'un des microARN les plus étudiés : il est impliqué dans la réponse immunitaire innée et adaptative, et dans de nombreuses pathologies liées à I'inflammation [8].

Certains microARN sont présents en plusieurs copies dans le génome, d'autres sous forme d'une copie unique. Comptetenu des duplications géniques successives ayant pris place chez les vertébrés, la présence d'un exemplaire unique d'un gène régulateur est annonciatrice d'effets de dose, et l'excès ou le défaut d'activité peut se révéler dommageable pour l'organisme. C'est le cas pour miR-155, dont l'activité inhibitrice des transcrits codant le facteur régulateur d'épissage $\mathrm{QKI}$ (quaking) ne s'exerce qu'aux plus faibles concentrations [9].

\section{Surexpression de miR-155 dans} les démences associées au SD

Les analyses histopathologiques de coupes réalisées sur des cerveaux de patients montrent que des plaques séniles sont présentes dans $93 \%$ des démences associées à la maladie d'Alzheimer, mais uniquement dans $25 \%$ des démences liées au SD [10]. Dans les deux cas, la protéine Tau hyperphosphorylée est détectée sur $90 \%$ des coupes analysées. Les microARN du chromosome 21 , et tout particulièrement miR-155, sont surexprimés dans les démences liées au SD, ce qui s'accompagne d'une réduction concomitante de l'expression des gènes cibles de miR-155 comme $B A C H 1$ (répresseur transcriptionnel) et $B C L 6$ (facteur pro-survie) (Figure 1), ou encore CoRESTI ${ }^{2}, B I M, C Y C L I N D$ et SAPK4, qui présentent tous des fonctions neuronales. En particulier, CoRESTl est un répresseur de transcription impliqué dans le contrôle de la différencia-

\footnotetext{
${ }^{2}$ CORESTl appartient à un complexe corépresseur de Sumo$2 / 3$. La protéine BIM interprète et transmet des signaux de mort par apoptose. La cycline D contrôle la transition Gl-S du cycle cellulaire. SAPK4 est une sérine-thréonine kinase également nommée mitogen-activated protein kinase P38-
} delta. tion neuronale, et son inhibition a pour résultat d'empêcher cette différenciation. Ceci explique peut-être en partie la perte de mémoire liée à l'impossibilité de mettre en place de nouveaux neurones au niveau de l'hippocampe dans les démences SD et MA. L'expression de ces différents gènes dans les démences SD est très généralement inférieure à ce qu'elle est dans les démences MA. Néanmoins, les gènes du chromosome 21 qui ne sont pas ciblés par miR-155, comme APP (amyloid precursor protein) et DYRKI (dual-specificity tyrosine phosphorylated-regulated kinase $1 A)$, ne sont pas surexprimés dans les démences SD, et leurs produits ne sont pas co-localisés avec la protéine Tau hyperphosphorylée, ce qui indique que des effets indépendants de miR-155 s'exercent également. Curieusement, on observe une surexpression à la fois de gènes pro-apoptotiques et anti-apoptotiques dans les démences MA et SD [10].

Pris dans leur globalité, ces résultats suggèrent que c'est plus la présence de Tau hyperphosphorylée que l'accumulation des plaques séniles qui joue le rôle principal dans l'apparition des démences, et que les anomalies moléculaires présentes dans les démences SD, dont certaines liées à la surexpression de miR-155 et à ses effets dose-dépendants, ne correspondent qu'imparfaitement à celles des démences MA même si elles s'accompagnent de symptômes similaires.

Le développement de stratégies de traitement spécifiques pour ralentir ou, à terme, empêcher l'apparition des démences chez les SD sera donc nécessaire.

Finalement, miR-155 joue également un rôle clé dans les paralysies consécutives aux opérations de réparation des anévrismes de l'aorte thoraco-abdominale [11]. Dans les cellules endothéliales, miR-155 cible les transcrits codant MFSD2 $A^{3}$, un facteur impliqué

\footnotetext{
${ }^{3}$ MFSD2A : major facilitator superfamily domain containing 2.
}

dans la stabilisation de la barrière hémato-encéphalique et dans le transport de l'acide docosahexaénoïque (DHA) vers les neurones. La concentration en DHA étant particulièrement importante au niveau des synapses, et cet acide gras étant le précurseur de molécules anti-inflammatoires, le rôle délétère de miR-155 dans les démences MA et surtout SD pourrait donc avoir pour origine le ciblage des transcrits MFSD2A par miR-155. Les recherches futures sur le rôle de miR-155 dans les neuropathologies devraient permettre le développement de nouvelles stratégies thérapeutiques ciblant ce microARN. $\diamond$

Roles of miR-155 microRNA in dementia associated with Down's syndrome

\section{LIENS D'INTÉRÊT}

Les auteurs déclarent n'avoir aucun lien d'intérêt concernant les données publiées dans cet article.

\section{RÉFÉRENCES}

1. Nieuwenhuis-Mark RE. Diagnosing Alzheimer's dementia in Down syndrome: problems and possible solutions. Res Dev Disabil 2009 ; 30 : 827-38.

2. Delabar JM. Le syndrome de Down - Nouvelles perspectives thérapeutiques? Med Sci (Paris) 2010 ; $26: 371-6$.

3. Zis P, Strydom A. Clinical aspects and biomarkers of Alzheimer's disease in Down syndrome. Free Radic Biol Med 2018; $114: 3-9$.

4. Ballard C Mobley W Hardy J, et al. Dementia in Down's syndrome. Lancet Neurol 2016; 15 : 622-36.

5. Pujol J, Fenoll R, Ribas-Vidal, et al. A longitudinal study of brain anatomy changes preceding dementia in Down syndrome. Neuroimage Clin 2018; 18 : 160-6.

6. Quinlan S, Kenny A, Medina M, et al. MicroRNAs in Neurodegenerative Diseases. Int Rev Cell Mol Biol 2017 ; 334 : 309-43.

7. Brás B, Rodrigues AS, Gomes B, Rueff J. Down syndrome and microRNAs. Biomed Rep 2018 ; 8 : 116.

8. Tili $\varepsilon$, Michaille J-J, Croce CM. MicroRNAs play a central role in molecular dysfunctions linking inflammation with cancer. Immunol Rev $2013 ; 253$ : 167-84

9. Tili $\varepsilon$, Chiabai M, Palmieri D, et al. Quaking and miR155 interactions in inflammation and leukemogenesis. Oncotarget $2015 ; 6: 24599-610$.

10. Tili $\varepsilon$, Mezache L, Michaille J-J, et al. microRNA 155 up regulation in the CNS is strongly correlated to Down's syndrome dementia. Ann Diagn Pathol 2018 ; 34 : 103-9.

11. Awad H, Bratasz A, Nuovo G, et al. MiR-155 deletion reduces ischemia-induced paralysis in an aortic aneurysm repair mouse model: Utility of immunohistochemistry and histopathology in understanding etiology of spinal cord paralysis. Ann Diagn Pathol 2018 ; 36 : 12-20. 